\title{
Optimally Placing Photovoltaic Systems in Distribution Networks Considering the Influence of Harmonics on Power Losses
}

\author{
Anh Tuan Doan ${ }^{1}$, Minh Quan Duong ${ }^{1 *}$ and Marco Mussetta ${ }^{2}$ \\ ${ }^{1}$ Department of Electrical Engineering, University of Science and Technology, \\ The University of DaNang, 54 Nguyen Luong Bang Street, LienChieu District, Vietnam \\ ${ }^{2}$ Politecnico di Milano, Dipartimento di Energia, Via La Masa 34, 20156 Milano, Italy \\ dmquan@dut.udn.vn,datuan@ac.udn.vn,Marco.Musetta@polimi.it \\ *Corresponding author: dmquan@dut.udn.vn
}

\begin{abstract}
Fossil fuels cause many negative impacts on the environment. Thus, the integration of renewable energy sources into traditional systems is increasingly prioritized for development. In this paper, Improved Coyote Optimization Algorithm (ICOA) is proposed for finding the most suitable location and the best capacity of photovoltaic distributed generators (PDGs) in radial distribution systems (RDSs). This new algorithm is an upgraded version of Coyote Optimization Algorithm (COA) and it has a better performance, a faster convergence speed and a more stable feature than its original form. The study focuses on the main objective function of minimizing both total active power losses on conductors and the impact of harmonics on node voltage and current flowing in distribution lines. In addition to currents with base frequency, harmonic flows also cause active power loss in distribution lines and the negative impact is also considered in the paper as a novelty. ICOA together with COA and Salp Swarm Algorithm (SSA) are applied for the IEEE 69-node radial distribution system for comparison with other methods in the literature. The comparative analysis sees that ICOA is a powerful method in optimally placing PDGs for reducing power loss and harmonic distortions.
\end{abstract}

Keywords: Coyote optimization algorithm; photovoltaic distributed generator; power losses; harmonic distortions; distribution lines.

Nomenclature
$\operatorname{CtrlV}_{v}^{\text {Min }}, C \operatorname{trl} V_{v}^{\text {Max }}$
$\operatorname{CtrlV}_{v, c o, p a}^{\text {New }}$
$C, C^{\text {Max }}$
$\Delta V_{B s, c o, p a}$
$\Delta I_{B h, c o, p a}$
$\Delta T H D_{B s, c o, p a}$
$\Delta I H D_{B S, c o, p a}^{h}$
$F F_{c o, p a}$
$F F_{c o, p a}^{N e w}$
$F F_{b, p a}$
$F F_{a, p a}$
$F F_{\text {mean }, p a}$
$F F_{L b e s t, p a}$
$H$
$I_{B h, P D G}$

The lowest and highest values of the $v^{\text {th }}$ control variable The new value of the $v^{\text {th }}$ control variable in the $c o^{\text {th }}$ solution in $p a^{\text {th }}$ pack

The current and maximum values of pairs of close solutions The voltage, $\mathrm{y}$ at the $B s^{\text {th }}$ node corresponding to the $c o^{\text {th }}$ solution in the $p a^{\text {th }}$ pack

The current violation penalty for the $B h^{\text {th }}$ branch corresponding to the $c o^{\text {th }}$ solution in the $p a^{\text {th }}$ pack

The total harmonic distortion violation penalty at the $B s^{\text {th }}$ node corresponding to the $c o^{\text {th }}$ solution in the $p a^{\text {th }}$ pack The individual harmonic distortion violation penalty at the $B s^{\text {th }}$ node corresponding to the $c o^{\text {th }}$ solution in the $p a^{\text {th }}$ pack The current fitness value at the $c o^{\text {th }}$ solution in the $p a^{\text {th }}$ pack The new fitness value at the $c o^{\text {th }}$ solution in the $p a^{\text {th }}$ pack The fitness value of $b^{\text {th }}$ solution in the $p a^{\text {th }}$ pack The fitness value of $a^{\text {th }}$ solution in the $p a^{\text {th }}$ pack The average fitness value of solutions in the $p a^{\text {th }}$ pack The best fitness value in the $p a^{\text {th }}$ pack The maximum number of the order harmonic The current magnitude at the $B h^{\text {th }}$ branch after connecting PDGs

Received: March $10^{\text {th }}, 2021$. Accepted: April $30^{\text {th }}, 2021$

DOI: 10.15676/ijeei.2021.13.2.1 


\begin{tabular}{|c|c|}
\hline$I_{B h}^{1}, I_{B h, P D G}^{1}$ & $\begin{array}{l}\text { The current magnitude at the fundamental frequency before } \\
\text { and after integrating PDGs }\end{array}$ \\
\hline$I_{B h}^{h}, I_{B h, P D G}^{h}$ & $\begin{array}{l}\text { The current magnitude at the higher order frequency before } \\
\text { and after integrating PDGs }\end{array}$ \\
\hline$I H D_{V, B S}^{h}$ & $\begin{array}{l}\text { The individual voltage harmonic distortion at the } h^{\text {th }} \text { order } \\
\text { harmonic of the } B s^{\text {th }} \text { node }\end{array}$ \\
\hline$I^{\operatorname{Max}}$ & The maximum current magnitude limit \\
\hline$I H D^{\operatorname{Max}}$ & The maximum limit of the individual harmonic distortion \\
\hline$I_{B h, c o, p a}$ & $\begin{array}{l}\text { The current magnitude at the } B h^{\text {th }} \text { branch corresponding to } \\
\text { the } c o^{\text {th }} \text { solution in the } p a^{\text {th }} \text { pack }\end{array}$ \\
\hline$I H D_{B S, c o, p a}^{h}$ & $\begin{array}{l}\text { The individual harmonic distortion values at the } B s^{\text {th }} \text { node } \\
\text { corresponding to the } c o^{\text {th }} \text { solution in the } p a^{\text {th }} \text { pack }\end{array}$ \\
\hline$I t, I t^{\operatorname{Max}}$ & $\begin{array}{l}\text { The current number of iteration and the maximum number } \\
\text { of iteration }\end{array}$ \\
\hline$N_{B h}$ & The total branch number \\
\hline$N_{P D G}$ & The total photovoltaic distributed generator number \\
\hline$N_{L d}$ & The total load number \\
\hline$N_{c o}$ & The coyote number in the community \\
\hline$N_{p a}$ & The coyote pack number in the community \\
\hline$N_{B s}$ & The total bus number \\
\hline$N_{v}$ & The total control variable number \\
\hline$N_{p o}$ & The number of coyote population \\
\hline$O F_{A}$ & The first component value in the multi-objective function \\
\hline$O F_{B 1}$ & $\begin{array}{l}\text { The first part value in the second component of the multi- } \\
\text { objective function }\end{array}$ \\
\hline$O F_{B 2}$ & $\begin{array}{l}\text { The second part value in the second component of the multi- } \\
\text { objective function }\end{array}$ \\
\hline$O F_{B}$ & The second component value in the multi-objective function \\
\hline$O F_{c o, p a}$ & $\begin{array}{l}\text { The objective function value at the } c o^{\text {th }} \text { solution in the } p a^{\text {th }} \\
\text { pack }\end{array}$ \\
\hline$P_{L S}, P_{L S, P D G}$ & $\begin{array}{l}\text { The total active power loss before and after integrating } \\
\text { PDGs }\end{array}$ \\
\hline $\mathrm{P}_{\mathrm{Ls}, \mathrm{PDG}, 1}$ & $\begin{array}{l}\text { The active power loss at the } B h^{\text {th }} \text { branch after integrating } \\
\text { PDGs }\end{array}$ \\
\hline$P_{G r}$ & The total active power from substation \\
\hline$P_{L d, l}$ & The total active power of loads at the $l^{\text {th }}$ load \\
\hline$P_{P D G, p}$ & The capacity of PDG at the $p^{\text {th }}$ PDG \\
\hline$P_{w}$ & The control parameter in the extra algorithm \\
\hline$P_{P D G}^{M i n}, P_{P D G}^{M a x}$ & The minimum and maximum capacity of PDG \\
\hline$R_{B h}$ & The resistance value at the $B h^{\text {th }}$ branch in the system \\
\hline$r d, r d_{1}, r d_{2}, r d_{3}$ & The numbers are generated in the range of $[0,1]$ \\
\hline$\rho$ & The penalty factor in the fitness objective function \\
\hline$S_{c o, p a}$ & The current solution of the $c o^{\text {th }}$ solution in the $p a^{\text {th }}$ pack \\
\hline$S^{\operatorname{Min}}, S^{\operatorname{Max}}$ & The minimum and maximum limits of solution \\
\hline$S_{c o, p a}^{N e w}$ & The new solution of the $c o^{\text {th }}$ solution in the $p a^{\text {th }}$ pack \\
\hline$S_{\text {Lbest,pa }}$ & The best solution in the $p a^{\text {th }}$ pack \\
\hline$S_{r 1, p a}, S_{r 2, p a}$ & The solutions which are randomly taken in the $p a^{\text {th }}$ pack \\
\hline$S_{\text {Gbest }}$ & The best solution in the population \\
\hline$S_{\text {Lbest }, 1}, S_{\text {Lbest }, 2}, S_{\text {Lbest }, 3}$ & $\begin{array}{l}\text { The best solutions which are randomly taken from different } \\
\text { packs }\end{array}$ \\
\hline$T H D_{V, B S}$ & The total voltage harmonic distortion at the $B s^{\text {th }}$ node \\
\hline
\end{tabular}


$T H D_{V, B S, P D G}$

$T H D_{B s, c o, p a}$

$V_{B S}^{h}$

$V_{B S}^{1}$

$V^{M i n}, V^{M a x}$

$V_{B S, P D G}$

$V_{B S, P D G}^{1}$

$V_{B s, c o, p a}$
The total voltage harmonic distortion at the $B u^{\text {th }}$ node after integrating PDGs

The total harmonic distortion values at the $B s^{\text {th }}$ node corresponding to the $c o^{\text {th }}$ solution in the $p a^{\text {th }}$ pack

The voltage magnitude for the $h^{\text {th }}$ order harmonic at the the $B s^{\text {th }}$ node

The voltage magnitude for the fundamental frequency at the $B s^{\text {th }}$ node

The minimum and maximum voltage magnitude limits The total voltage magnitude at fundamental and higher order frequencies of the $B s^{\text {th }}$ node after integrating PDGs

The fundamental voltage magnitude at the $B s^{\text {th }}$ node after integrating PDGs

The node voltage at the $B s^{\text {th }}$ node corresponding to the $c o^{\text {th }}$ solution in the $p a^{\text {th }}$ pack

Abbreviations

$\mathrm{ABC}$

Artificial Bee Colony

AGA

Adaptive Genetic Algorithm

ALOA

Ant Lion Optimization Algorithm.

BBO

Biogeography Based Optimization

BFOA

Bacterial Foraging Optimization Algorithm

COA

Coyote Optimization Algorithm

FPA

Flower Pollination Algorithm

GA

Genetic

Algorithm.

HAS

Harmony Search Algorithm

ICOA

Improved Coyote Optimization Algorithm

IWO

Invasive Weed Optimization Algorithm

PBIL

Population-based Incremental Learning Algorithm

PSO

Particle Swarm Optimization

QOTLBO

Quasi-Oppositional Teaching Learning Based Optimization

SA Simulated Annealing

SCA

Sine Cosine Algorithm

SSA

Salp Swarm Algorithm 
Anh Tuan Doan, et al.

WOA

Whale Optimization Algorithm

\section{Introduction}

In order to minimize the impact of fossil energy sources on the environment, renewable energy sources are increasingly developing in most countries around the world. The connection of renewable energy sources such as wind energy, solar energy, geothermal energy, ocean wave energy, etc. must be carefully considered. Many researchers have focused on analyzing the effects of this grid integrated generator system to maximize benefits [1-2]. The integration can bring many benefits to the distribution systems such as improving voltage of loads, mitigating harmonic distortions, reducing power loss on the transmission lines, minimizing emissions and reducing operating costs [3]. However, many studies have shown that improper connection of these distributed sources can cause undesirable effects and negatively impact on the economy, technology and environment [4]. Thus, researchers focused on determining the optimal installation of distributed generators (DGs) in the distribution systems. In Ref. [5], authors proposed ALOA for searching the optimal location and capacity of single and multiple renewable distributed generations. Their solution has succeeded in minimizing the power of loss and maximizing saving cost of energy loss in the radial distribution systems. Besides, the authors in Ref. [6] also reduced total power loss on the transmission lines by applying GA as an optimization tool with multiple placements of DGs. By determining the suitable installation of DGs, the total power loss is significantly reduced in a test case, IEEE 14-node network. For the same target of the loss reduction maximization, the study [7] used FPA to determine the appropriate capacity and suitable placement of photovoltaic - based distributed generation in distribution system with 41 nodes. The results showed the positivity of the solution in maximizing the benefits. In addition to reducing losses, many studies have tried to achieve the high voltage stability by adding DGs into distribution systems [8-11]. As shown in the numerical results, the reduction of the power loss and the improvement of voltage stability were highly effectively after connecting DGs by using AGA, SA, QOTLBO and ABC, respectively. Additionally, in Refs. [12-13], analytical method and HSA have also been used for determining the appropriate integration strategy of DGs in the aim of the loss reduction and voltage improvement for IEEE 33-node and 69-node systems. Those authors indicated the strong impact of the DGs installation on the economic and technical evaluations of a distribution system. With the desire to consider comprehensively the optimization problem of DGs, the authors in Refs. [14-15] proposed BFOA and IWO respectively for reducing of power loss as well as operating costs and improving voltage profile in the distribution system. The results have proven that the integration of DGs into grid not only improved the technical issues in the system but also helped to save costs during operation. With the same test systems and objective functions, the authors in Refs. [16-18] proposed strong hybrid methods such as WOA-SCA, PBIL-PSO and GA/PSO, respectively for solving the optimization problem of integrating DGs. The efficiency of these hybrid methods has been proven by comparing the obtained solutions. Furthermore, another research case in Ref. [19] has focused on the study for reliability enhancement by connecting of DGs. These authors have used an average index of the interruption frequency, the expected interruption cost and the interruption duration for analyzing reliability indices and losses. That paper demonstrated that the reliability of the system can be enhanced thanks to the selection of suitable installation of DGs.

In general, almost all previous studies only focused on improving voltage quality and minimizing power loss. They have not concerned another important element of harmonic flows. For distribution systems with nonlinear loads, the existence of harmonics is inevitable and it can negatively affect the electric device if values of the total harmonic distortion and the individual harmonic distortion exceed allowed limits. Thus, a high number of researchers has identified the danger of harmonics and suggested adding DGs in distribution systems to avoid the unintentional consequence [20-22]. Specifically, BBO [20] and sensitivity analysis [21] have been respectively applied for installing DGs to mitigate the average value of the total voltage harmonic distortion. 
Nonlinear loads have been added in the distribution systems in charge of generating harmonic flows with different amplitudes and different orders. Power flows on distribution lines were still solved by using the forward/backward sweep technique (FW/BWST) similarly as the systems without nonlinear loads. Besides, other authors in Ref. [22] have considered for minimizing harmonics as an objective function under many constraints of node voltage, harmonics, sizing and penetration limits. Clearly, by using the optimization algorithm for searching the optimal position and capacity of multiple DGs, harmonics have been drastically reduced and obtained results have satisfied IEEE Std. 519 exactly. However, most of the studies that have harmonic analysis calculated only loss and voltage values at the fundamental frequency. Realistically, losses and voltages persist at the higher order frequency [23]. Therefore, it is important to study and compute numerical results at frequency orders to obtain exact solutions.

In this paper, Improved Coyote Optimization Algorithm (ICOA) [24] is applied as an optimal method for determining the suitable location and capacity of Photovoltaic Distributed Generators in IEEE 69-node radial distribution system. ICOA is an updated version of COA, so it has inherited the outstanding features of COA and the improvements to the original algorithm will greatly contribute to enhancing the performance and stability of the algorithm. The main goal is to minimize a bi-objective function including the power loss and the harmonic distortions under the multiple constraints. In the power loss computation process, the impact of all harmonics on the power loss of all conductors is considered unlikely previous studies that only considered the fundamental frequency current. In terms of harmonic objective, total harmonic distortion and individual harmonic distortion are calculated on average and the result become the second objective in the bi-objective function of the problem. On the other hands, basic constraints of distribution systems and additional advanced constraints regarding harmonics are also taken into account in the study. The increase of voltage amplitude due to the impact of harmonics is also calculated and added to voltage of loads at the fundamental frequency. In summary, the novelties of the paper are as follows:

1. Consider harmonics' influence on power loss of conductors. The power loss is also a part of bi-objective function and considered in the power balance constraint.

2. Consider harmonics' influence on load voltage. Voltage amplitude of loads, which is increased due to the influence of harmonics, is constrained within an allowable range of distribution systems.

3. Apply SSA, COA and ICOA for the problem with new proposals in objective function and constraints.

The main contributions of this study can be briefly summarized into three main points as follows:

1. Proposed a novel method with high efficiency (ICOA) in finding the optimal solution for the position and capacity of PDGs in the system to maximize benefits. The obtained results will be compared with implemented methods and published methods to prove the superiority of the proposed method.

2. Analyzed the effects of PDGs when integrated into the distribution system, IEEE 69-node system.

3. Considered reduction of losses and harmonics in the system with nonlinear loads. Besides, the values of loss and voltage are calculated in the frequency orders to obtain the correctly numerical results.

The remaining contents of the paper are organized as follows: Sec. 2 presents the objective function and the constraints of the considering problem, call is the problem formulation. Sec. 3 introduces the proposed method and describes the application of the method to find the optimal location and capacity of PDGs in the distribution system, name is the proposed method. Sec. 4 shows all results obtained from the simulation and makes the discussion, call is the simulation results and discussion. The last section is the summary for the whole contents of this paper, name is conclusions. 


\section{Problem Formulation}

\section{A. The Objective function}

In this study, power losses and harmonic flows are important factors in evaluating the efficiency and power quality of the distribution system. Hence, they are considered as two main components in the multi-objective function of the problem. The two single objectives are expressed in detail as follows:

\section{A.1. The first single objective-Total power loss}

The power loss reduction is considered as a member of objective function due to the advantage of economic benefits. If power loss reduction is effectively finished, energy loss in $\mathrm{kWh}$ and energy loss cost in $\$$ are also significantly optimized over scheduled horizon. Therefore, power losses on the transmission lines should be taken seriously and become an evaluation criterion of connecting PDGs in the distribution systems [25]. The calculation of the total power loss after and before connecting PDGs is shown in Eq. (1) and Eq. (2) [20, 23], respectively.

$$
\begin{aligned}
& P_{L S, P D G}=\sum_{B h=1}^{N_{B h}}\left(I_{B h, P D G}\right)^{2} \cdot R_{B h}=\sum_{B h=1}^{N_{B h}}\left(\sqrt{\left|I_{B h, P D G}^{1}\right|^{2}+\sum_{h=2}^{H}\left|I_{B h, P D G}^{h}\right|^{2}}\right)^{2} \cdot R_{B h} \\
& P_{L S}=\sum_{B h=1}^{N_{B h}}\left(I_{B h}\right)^{2} \cdot R_{B h}=\sum_{B h=1}^{N_{B h}}\left(\sqrt{\left|I_{B h}^{1}\right|^{2}+\sum_{h=2}^{H}\left|I_{B h}^{h}\right|^{2}}\right)^{2} \cdot R_{B h}
\end{aligned}
$$

To evaluate the fitness function simply, the components in general as well as the first component in the multi-objective function will be kept within the range of 0 to 1 . Clearly, the first component associated with the power loss is always expected to be minimum and it is mathematically formed as follows:

$$
\text { Minimize } O F_{A}=\frac{P_{L S, P D G}}{P_{L S}}
$$

\section{A.2. The second single objective-Harmonic distortions}

Harmonic flows make the change of voltage wave form and also increase the voltage magnitude. These harmonic flows have different orders and different magnitudes that considerably influence voltage of loads. Each harmonic order has individual impact on the voltage of loads and all harmonic orders cause a stronger impact on the voltage. So, the individual impact of each harmonic order and the general impact of all harmonic orders must be considered in the load voltage quality evaluation. As a result, the total voltage harmonic distortion $\left(T H D_{V, B s}\right)$ caused by all harmonic orders and the individual voltage harmonic distortion at the higher order frequency $\left(I H D_{V, B S}^{h}\right)$ are considered as the second component of the multi-objective function. $T H D_{V, B s}$ and $I H D_{V, B S}^{h}$ are obtained by using the two following formulas [26].

$$
\begin{aligned}
& T H D_{V, B S}(\%)=\frac{\sqrt{\sum_{h=2}^{H}\left|V_{B S}^{h}\right|^{2}}}{\left|V_{B S}^{1}\right|} \cdot 100 \\
& I H D_{V, B S}^{h}(\%)=\frac{\left|V_{B S}^{h}\right|}{\left|V_{B S}^{1}\right|} \cdot 100
\end{aligned}
$$

The second component consists of two parts, $T H D_{V, B s}$ and $I H D_{V, B S}^{h}$. In this study, those parts are considered for minimizing the impact of harmonics on the voltage of the distribution systems. Thus, we propose Eq. (6) and Eq. (7) to calculate representative values and to keep the second single objective within the range of 0 and 1 .

$$
\begin{aligned}
& O F_{B 1}=1-\frac{1}{e^{\max \left(T H D_{V, B S}\right)}} \\
& O F_{B 2}=1-\frac{1}{e^{\max \left(I H D_{V, B S}^{h}\right)}}
\end{aligned}
$$


Because the two parts have the same range within 0 and 1 and they have the same impact on the voltage quality, their mean is considered as the second singe objective in the bi-objective function of the problem. As a result, the second objective in formula (8) is written to calculate the equilibrium value for this second component.

$$
\text { Minimize } O F_{B}=\frac{O F_{B 1}+O F_{B 2}}{2}
$$

In this research, the multi-objective function is used to evaluate the quality of the solution and it is established as Eq. (9)

Minimize $O F=\min \left[\left(w_{A} . O F_{A}\right)+\left(w_{B} . O F_{B}\right)\right]$

Finally, in this case, the weight sum method is applied to make decision for the value of the multi-objective function. The weight factor $w_{A}$ associated with the first objective-power loss and the weight factor $w_{B}$ associated with the second objective-harmonic distortions are constrained as follows [27].

$$
0 \leq w_{A}, w_{A} \leq 1 \& w_{A}+w_{B}=1
$$

\section{B. Constraints}

\section{B.1. The power balance constraint}

In this case, the distribution system is integrated PDGs for maximum benefits. Thus, the power balance equation in this system can be presented as [18]

$$
P_{G r}=\sum_{l=1}^{N_{L d}} P_{L d, l}+\sum_{B h=1}^{N_{B h}} P_{L s, P D G, B h}-\sum_{p=1}^{N_{P D G}} P_{P D G, p}
$$

\section{B.2. The branch current constraint}

In order to keep the thermal component on the transmission line from being altered with the integration of the PDGs. The current magnitude at harmonic orders after connecting PDGs should not exceed the limit as [28]

$$
I_{B h, P D G} \leq I^{M a x}, B h=1,2, \ldots, N_{B h}
$$

\section{B.3. The harmonic voltage distortion constraints}

The harmonic distortions in the distribution system can damage the electronic equipment that connected on the transmission lines. Therefore, the harmonic values at nodes should comply with the IEEE Std. 519 about allowed limits.

The limit for the total voltage harmonic distortion [29]

$$
T H D_{V, B S}(\%) \leq T H D^{\operatorname{Max}}(\%)
$$

The limit for the individual voltage harmonic distortion [30]

$$
I H D_{V, B S}^{h}(\%) \leq I H D^{\operatorname{Max}}(\%)
$$

\section{B.4. The bus voltage limits}

The integration of PDGs can have a powerful effect on the distribution system, such as voltage profile, stability, reliability, protection, etc. Therefore, it is necessary to keep the total voltage magnitude at the harmonic orders PDGs within the allowable limits as below [28, 23]:

$$
\begin{aligned}
& V^{M i n} \leq\left|V_{B S, P D G}\right| \leq V^{M a x}, B u=1,2, \ldots, N_{B S} \\
& V_{B S, P D G}=V_{B S, P D G}^{1} \cdot \sqrt{1+\left(\frac{T H D_{V, B S, P D G}}{100}\right)}
\end{aligned}
$$

\section{B.5. The $P D G$ 's penetration limits}

In this paper, the penetration of each PDG is considered with variation within a predetermined limits and the total capacity should not exceed the total load demand in the distribution system. So, the penetration level is kept in the constraints as below [20, 31]:

$$
\begin{aligned}
& \sum_{p=1}^{N_{P D G}} P_{P D G, p} \leq \sum_{l=1}^{N_{L d}} P_{L d, l} \\
& P_{P D G}^{M i n} \leq P_{P D G, p} \leq P_{P D G}^{M a x}
\end{aligned}
$$




\section{The Proposed Method}

In this study, two enhancements in the Coyote Optimization Algorithm are implemented to solve the problem of optimization and called ICOA. ICOA is developed to improve the COA's efficiency as well as the speed of convergence [32]. In both COA and ICOA, the two main components that are represented for individual in the coyote community are the quality of social condition and the social condition. A social condition represents as an optimal solution, while the quality of the social condition corresponds to the fitness of the solution. In the applied algorithms, the number of coyote individuals in each group $\left(N_{c o}\right)$ and the number of coyote groups in the coyote community $\left(N_{p a}\right)$ are the two main determinants of population $\left(N_{p o}\right)$. Hence, the total population is the multiplication of $N_{p a}$ and $N_{c o}$.

As mentioned, the grid integrated PDGs system brings many economic and technical benefits. However, this integration can cause undesirable effects on the system. Hence, the calculation of power flow and harmonic flow when connecting PDGs should be calculated carefully. In this study, the flow analysis at the fundamental frequency and at the high order frequency is based on forward / backward sweep technique (FW / BWST) which is clearly described in [33] and the nonlinear loads in the system are also considered as injectors of harmonic currents. In FW / BWST, the relationship between the branch currents and the injected bus currents are built by backward sweep. Besides, the forward sweep is responsible for determining the bus voltages by using the aforementioned relationship in the distribution system. The process of using ICOA for finding the optimal solution of PDGs installation can be briefly summarized as below:

STEP 1: Determine the initial parameters such as $N_{c o}, N_{p a}$, and $I t^{\operatorname{Max}}$

STEP 2: Randomly produce the initial solution set in the predetermined limits of $\left[S^{M i n}, S^{M a x}\right]$ by using Eq. (19):

$$
S_{c o, p a}=S^{M i n}+r d .\left(S^{M a x}-S^{M i n}\right) ; c o=1,2, \ldots, N_{c o} \& p a=1,2, \ldots, N_{p a}
$$

STEP 3: Solve the power flow and harmonic flow by using the BW/FW sweep method [33]. Calculate the penalty level for the node voltages, the branch currents and the harmonics of solutions by applying Eq. (20) - Eq. (23), respectively:

$$
\begin{aligned}
& \Delta V_{B s, c o, p a}=\left\{\begin{array}{c}
\left|V^{M a x}-V_{B s, c o, p a}\right|, \text { if } V_{B s, c o, p a}>V^{M a x} \\
\left|V^{M i n}-V_{B s, c o, p a}\right|, \text { if } V_{B s, c o, p a}<V^{M i n} \\
0 \text { else }
\end{array}\right. \\
& \Delta I_{B h, c o, p a}=\left\{\begin{array}{c}
\left|I^{\text {Max }}-I_{B h, c o, p a}\right|, \text { if } I_{B h, c o, p a}>I^{\text {Max }} \\
\left|I^{\text {Min }}-I_{B h, c o, p a}\right|, \text { if } I_{B h, c o, p a}<I^{\text {Min }} \\
0 \text { else }
\end{array}\right. \\
& \Delta T H D_{B s, c o, p a}=\left\{\begin{array}{c}
\left|T H D^{M a x}-T H D_{B s, c o, p a}\right|, \text { if } T H D_{B s, c o, p a}>T H D^{M a x} \\
0 \text { else }
\end{array}\right. \\
& \Delta I H D_{B S, c o, p a}^{h}=\left\{\begin{array}{c}
\left|I H D^{M a x}-I H D_{B S, c o, p a}^{h}\right|, \text { if } I_{\text {IH }} D_{B S, c o, p a}^{h}>I H D^{M a x}, h>1 \\
0 \text { else }
\end{array}\right.
\end{aligned}
$$

Calculate the fitness value of solutions by using Eq. (24) and start the first computation iteration (i.e. set $I t=1$ )

$$
\begin{aligned}
& F F_{c o, p a}=O F_{c o, p a}+\rho \cdot\left(\sum_{B s=1}^{N_{B s}} \Delta V_{B s, c o, p a}+\sum_{B h=1}^{N_{B h}} \Delta I_{B h, c o, p a}+\right. \\
& \left.\sum_{B s=1}^{N_{B s}} \Delta T H D_{B s, c o, p a}+\sum_{B s=1}^{N_{B s}} \Delta I H D_{B s, c o, p a}^{h}\right)
\end{aligned}
$$

STEP 4: Determine and save the local best solution $\left(S_{\text {Lbest,pa }}\right)$ in each pack and the global best solution $\left(S_{\text {Gbest }}\right)$ in the current population. Produce new solutions by applying Eq. (25) below:

$$
\begin{aligned}
& S_{c o, p a}^{N e w}=S_{c o, p a}+r d_{1} \cdot\left(S_{L b e s t, p a}-S_{r 1, p a}\right)+r d_{2} \cdot\left(S_{G b e s t}-S_{r 2, p a}\right) \\
& c o=1,2, \ldots, N_{c o} \& p a=1,2, \ldots, N_{p a}
\end{aligned}
$$


STEP 5: In each generated new solution, the control variables of the new solutions are adjusted according to the predefined rules as Eq. (26)

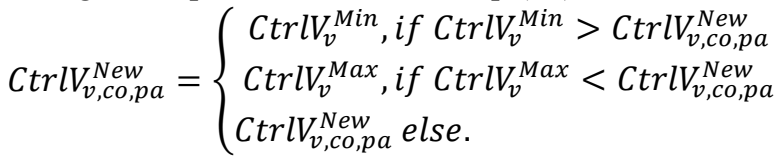

$$
\begin{aligned}
& v=1,2, \ldots, N_{v} ; c o=1,2, \ldots, N_{c o} ; p a=1,2, \ldots, N_{p a}
\end{aligned}
$$

STEP 6: Apply penalty functions at STEP 3 to compute the fitness value of the objective function for evaluating the quality of each solution by using Eq. (24).

STEP 7: Retain the solutions which have good quality by using Eq. (27) - Eq. (28)

$$
\begin{aligned}
\mathrm{S}_{c o, p a} & = \begin{cases}S_{c o, p a}^{N e w}, \text { if } F F_{c o, p a}>F F_{c o, p a}^{N e w} \\
\mathrm{~S}_{c o, p a} & \text { else }\end{cases} \\
\mathrm{FF}_{c o, p a} & = \begin{cases}F F_{c o, p a}^{N e w}, \text { if } F F_{c o, p a}>F F_{c o, p a}^{N e w} \\
\mathrm{FF}_{c o, p a} & \text { else }\end{cases}
\end{aligned}
$$

STEP 8: In this task, the second improvement is comprised of two update equations for generating a new solution. The selection of the update equation depends on the survey result of the control parameter $\left(P_{w}\right)$ within the range of $[0,1]$ to choose the suitable parameter and the calculation result of the proportional value $(R)$ of the two counters $\left(C\right.$ and $\left.C^{M a x}\right)$. As mentioned, it is important to determine the value of the counter $C$. Its value is changed when the difference of two adjacent fitness values is less than the difference of the mean and best values in the pack. Specifically, for each pack in the population, a new solution can be produced by using Eq. (29) or Eq. (30).

$$
\begin{aligned}
& S_{p a}^{\text {New }}=S_{\text {Gbest }}+r d_{1} \cdot\left(S_{\text {Gbest }}-S_{\text {Lbest }, 1}\right)+r d_{2} \cdot\left(S_{\text {Gbest }}-S_{\text {Lbest }, 2}\right) \\
& S_{\text {pa }}^{\text {New }}=S_{\text {Gbest }}+r d_{1} \cdot\left(S_{\text {Gbest }}-S_{\text {Lbest }, 1}\right)+r d_{2} \cdot\left(S_{\text {Gbest }}-S_{\text {Lbest }, 2}\right)+r d_{3} \cdot\left(S_{\text {Gbest }}-S_{\text {Lbest }, 3}\right)
\end{aligned}
$$

Besides, the $C^{\text {Max }}$ value is also calculated based on the number of coyotes such as Eq. (31)

$$
C^{\operatorname{Max}}=\frac{N_{c o} \times\left(1+N_{c o}\right)}{2}
$$

The calculation process to determine the condition for applying the update equation can be followed by the extra algorithm which is proposed in Ref. [24] as below box: 


$$
\begin{aligned}
& C=0 ; \\
& \text { For } a=1 \text { to }\left(N_{c o}-1\right) \\
& \text { For } b=a+1 \text { to } N_{c o} \\
& \text { If }\left|F F_{b, p a}-F F_{a, p a}\right|<\left|F F_{\text {mean }, p a}-F F_{\text {Lbest,pa }}\right| \\
& C=C+1
\end{aligned}
$$

End

End

End

Compute proportional value $\mathrm{R}$ as:

$$
R=\frac{C}{C^{\operatorname{Max}}}
$$

If $R>P_{w}$

Apply Eq. (29) to produce the new solution

Else

Apply Eq. (30) to produce the new solution

End;

After a new solution has been generated, each control variable in that solution is adjusted.

Run the power flow and harmonic flow.

Calculate the fitness value of the objective function after applying penalty terms.

STEP 9: Determine the best solution in population.

STEP 10: Check the condition in Eq. (34). If it is satisfied, exchange the solutions between the two randomly selected packs. Otherwise, check the criteria to exit the iteration as Eq. (35)

$$
\begin{aligned}
& r d<\frac{0.01}{2} . N_{c o}^{2} \\
& I t=I t^{\text {Max }}
\end{aligned}
$$

If the stopping criterion is not satisfied, come back to STEP 4 and the number of iterations is increased by one.

\section{The Simulation Results}

In this paper, ICOA is proposed for finding the location and capacity of three PDGs in the IEEE 69-node radial distribution system. This system has 69 nodes with the total load capacity of 3.8019 MW and 2.6941 MVar. The structure of the system is shown in Figureure 1. 


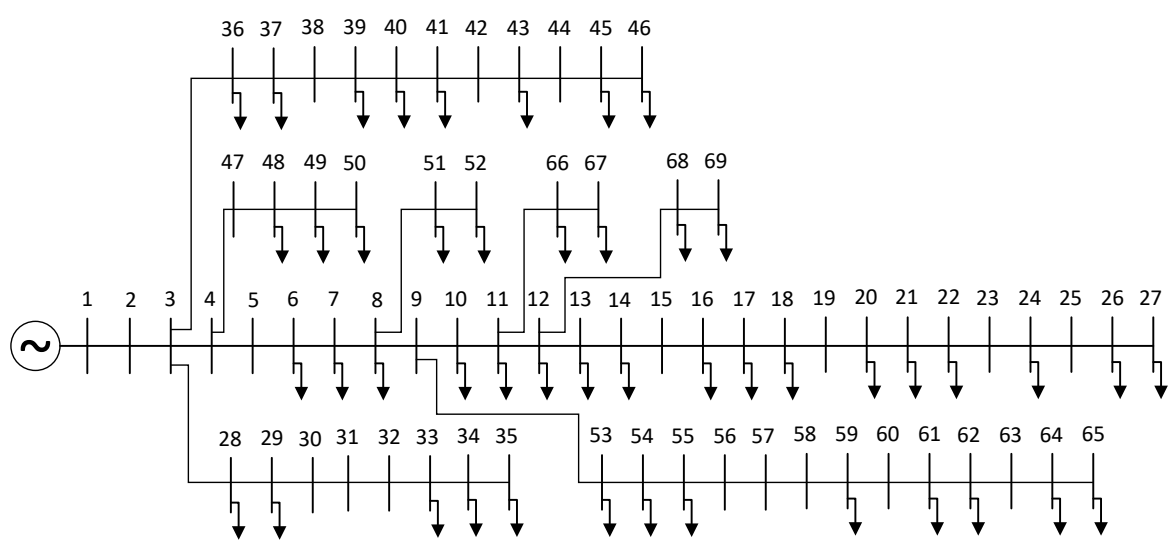

Figure 1. IEEE 69-node radial distribution system

As mentioned, this test system will be considered in the presence of harmonics. The harmonic flows whose magnitude, angle and harmonic order are shown in Figureure 2 are injected to load locations such as $10,12,18,19,22,25,31,38,46,56$, and 65 . According to the IEEE Std. 519, the maximum allowable limits of the total voltage harmonic distortion and the individual voltage harmonic distortion are $5 \%$ and $3 \%$, respectively. Besides, the voltage magnitude at each node should also be within the best range of $[0.95,1.05]$ (pu) [34].

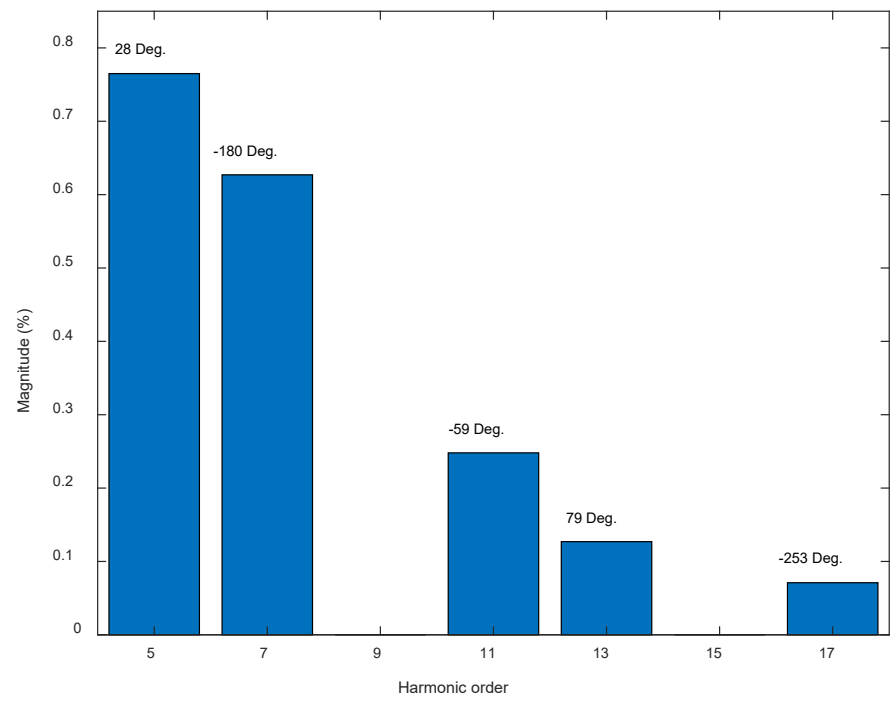

Figure 2. The detailed information of five harmonic flows

In the sum of weight method, the first factor $w_{A}$ is associated with the power loss reduction and the second factor $w_{B}$ is concerned with the harmonic mitigation. In this study, the minimization of both the power loss and harmonics is the main goal. However, the power loss reduction is assessed to be more important than harmonic reduction. Therefore, the first factor will have the value greater than the second factor. Specifically, $w_{A}=0.8$ and $w_{B}=0.2$. All simulations are implemented on personal computer by using MATLAB software. For personal computer specifications, Processor: $1.8 \mathrm{GHz}$ and RAM: $8.0 \mathrm{~GB}$. For the three implemented methods of SSA, COA and ICOA, the search region for the optimal location of each PDG will vary within the node limit of $[2,69]$ and the search capacity limit will also change in range of $[0.0,2.0](\mathrm{MW}) . I t^{\operatorname{Max}}$ is surveyed and selected is 100 with 60 trial runs $\left(\operatorname{Tr}^{\operatorname{Max}}\right)$ for each method. For running both COA and ICOA, $N_{c o}$ and $N_{p a}$ are surveyed for determining the most suitable 
value. In this particular case, the selected values of $N_{c o}$ and $N_{p a}$ should fall within the range of three numbers as 3,4 and 5. The value pairs of $N_{c o}$ and $N_{p a}$ are combined together and finally, the chosen result is 4 for $N_{c o}$ and $N_{p a}$. Besides, in ICOA, $P_{w}$ in the extra algorithm is taken as 0.2 [24]. Additionally, for SSA implementation, $N_{p o}$ is set to 20 for a fair comparison with three acceleration factors $\left(c_{1}, c_{2}\right.$ and $\left.c_{3}\right)$. While $c_{1}$ is the value of the function of $\left.2 e^{-\left(4 . I t / I t^{\text {Max }}\right.}\right)^{2}$, both $c_{2}$ and $c_{3}$ values are chosen randomly within the range of [0,1] [35].

Due to the characteristics of the meta-heuristic algorithms, 60 trial runs are performed for three implemented methods with randomly generated initial population. The obtained results are re-sorted in the increasing order of fitness function $t$ and presented as shown in Figure 3.

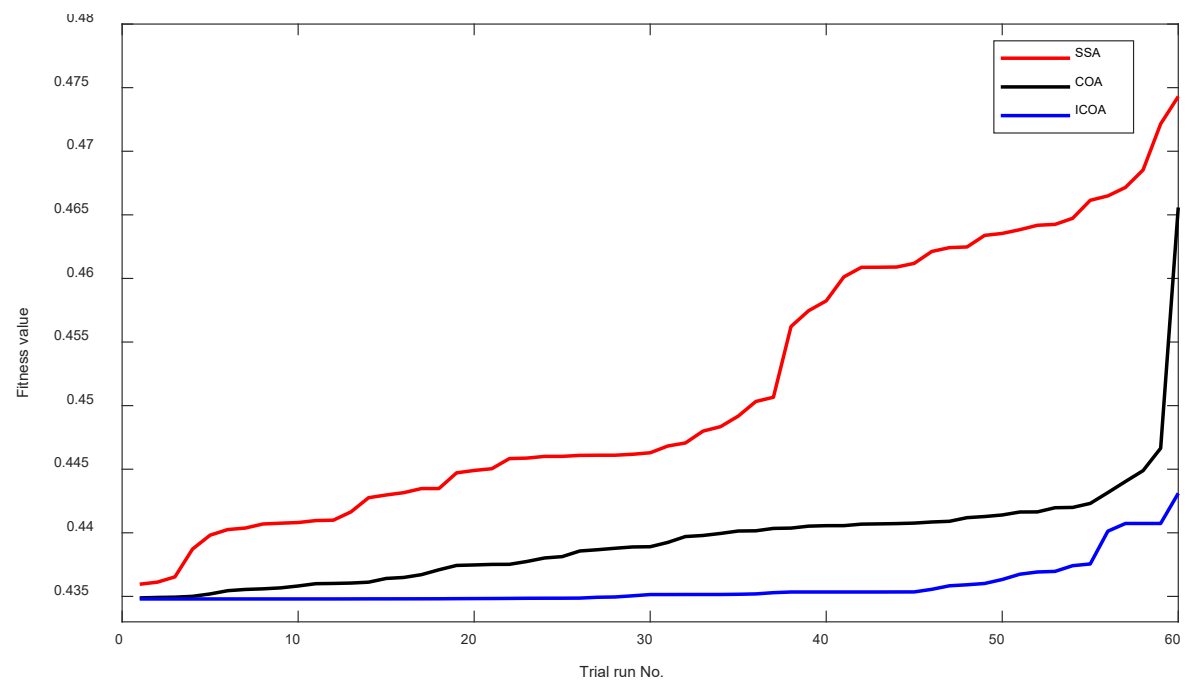

Figure 3. The re-sorted fitness values in order from smallest to biggest in 60 trial runs

By using the calculated results from Eq. (24), the worst fitness and the best fitness values are determined and the average fitness values of applied methods are computed in the 60 trials due to the stochastic characteristic of the heuristic algorithms. As shown in Table 1, the best fitness and the average fitness values of ICOA, COA and SSA are 0.4348 and $0.4358,0.4350$ and $0.4391,0.4360$ and 0.4513 , respectively. Obviously, the best fitness value of ICOA is the lowest as compared to others. This shows that the optimal solution from ICOA has the highest quality. Additionally, the average fitness value of ICOA is also lower than COA and SSA. This value represents the stability of each method and the closer it is to the best fitness value, the more stable the method is. In other words, ICOA is more stable than others. Besides, the worst fitness value of ICOA in 60 trials is 0.4431 and it is the lowest value as compared to COA and SSA, 0.4467 and 0.4743 , respectively. All the collected results have contributed to prove the high efficiency and stability of the proposed method. In addition, the number of fitness function evaluation is also calculated as the multiplication of the number of iterations and the population size in each trial run. This value of ICOA is smaller than SSA and equal to COA. In other words, ICOA uses fewer solutions to find the optimal solution. This saves data memory. All the above arguments have indicated that ICOA has a better stability and a stronger search feature than others in solving the same optimization problem. 
Table 1. The best, the mean and the worst fitness values of the implemented methods in 60 trial runs

\begin{tabular}{|l|c|c|c|c|}
\hline Method & $\begin{array}{c}\text { The worst } \\
\text { fitness value }\end{array}$ & $\begin{array}{c}\text { The average } \\
\text { fitness value }\end{array}$ & $\begin{array}{c}\text { The best } \\
\text { fitness value }\end{array}$ & $\begin{array}{c}\text { The number of } \\
\text { solution evaluations }\end{array}$ \\
\hline SSA & 0.4743 & 0.4513 & 0.4360 & 2000 \\
\hline COA & 0.4467 & 0.4391 & 0.4350 & 1600 \\
\hline ICOA & 0.4431 & 0.4358 & 0.4348 & 1600 \\
\hline
\end{tabular}

Table 2. The first, the second and the total objective function values of the implemented methods at the best solution

\begin{tabular}{|c|c|c|c|}
\hline Method & $O F_{A}$ & $O F_{B}$ & $O F$ \\
\hline SSA & 0.3193 & 0.9027 & 0.4360 \\
\hline COA & 0.3129 & 0.9235 & 0.4350 \\
\hline ICOA & 0.3126 & 0.9235 & 0.4348 \\
\hline
\end{tabular}

Table 3. The comparison of loss reduction of the implemented methods and the published methods at the best solution

\begin{tabular}{|c|c|c|c|c|}
\hline Method & $\begin{array}{l}\text { The optimal solution of three PDGs } \\
\text { (Place - Capacity) }\end{array}$ & $\begin{array}{l}\text { Power loss } \\
\text { (MW) }\end{array}$ & $\begin{array}{l}\text { Populatio } \\
\text { n size }\end{array}$ & $\begin{array}{c}\text { Iteration } \\
\text { number }\end{array}$ \\
\hline $\begin{array}{l}\text { GA/PSO } \\
{[18]}\end{array}$ & $\begin{array}{c}\text { Node } 21-0.9105 \text { MW; Node } 61-1.1926 \\
\text { MW; Node } 63-0.8849 \text { MW }\end{array}$ & 0.0811 & 50 & 30 \\
\hline $\begin{array}{l}\text { SA } \\
{[9]}\end{array}$ & $\begin{array}{c}\text { Node } 18-0.4204 \text { MW; Node } 60-1.3311 \\
\text { MW; Node } 65-0.4298 \text { MW }\end{array}$ & 0.0771 & 30 & 50 \\
\hline $\begin{array}{l}\text { BFOA } \\
{[14]}\end{array}$ & $\begin{array}{c}\text { Node } 27-0.2954 \text { MW; Node } 61-1.3451 \\
\text { MW; Node } 65-0.4476 \text { MW }\end{array}$ & 0.0752 & 100 & 50 \\
\hline $\begin{array}{l}\text { HSA } \\
{[13]}\end{array}$ & $\begin{array}{c}\text { Node } 63-1.3024 \text { MW; Node } 64-0.3690 \\
\text { MW; Node } 65-0.1018 \text { MW }\end{array}$ & 0.0868 & 30 & 60 \\
\hline $\begin{array}{l}\text { IWO } \\
{[15]}\end{array}$ & $\begin{array}{c}\text { Node } 27-0.2381 \text { MW; Node } 61-1.3266 \\
\text { MW; Node } 65-0.4334 \text { MW }\end{array}$ & 0.0746 & 100 & 50 \\
\hline $\begin{array}{l}\text { QOTLBO } \\
{[10]}\end{array}$ & $\begin{array}{c}\text { Node } 15-0.8114 \mathrm{MW} \text {; Node } 61-1.1470 \\
\text { MW; Node } 63-1.0020 \mathrm{MW}\end{array}$ & 0.0806 & 50 & 100 \\
\hline $\begin{array}{l}\text { AGA } \\
{[8]}\end{array}$ & $\begin{array}{c}\text { Node } 12-0.2720 \mathrm{MW} \text {; Node } 21-0.3100 \\
\text { MW; Node } 61-1.8610 \mathrm{MW}\end{array}$ & 0.0707 & 80 & 300 \\
\hline SSA & $\begin{array}{c}\text { Node } 17-0.4118 \mathrm{MW} \text {; Node } 61-1.5519 \\
\text { MW; Node } 64-0.2886 \mathrm{MW}\end{array}$ & 0.0719 & 20 & 100 \\
\hline $\mathrm{COA}$ & $\begin{array}{l}\text { Node } 11-0.5056 \mathrm{MW} \text {; Node } 17-0.3791 \\
\text { MW; Node } 61-1.6904 \mathrm{MW}\end{array}$ & 0.0705 & 16 & 100 \\
\hline ICOA & $\begin{array}{c}\text { Node } 11-0.4883 \mathrm{MW} \text {; Node } 17-0.3863 \\
\text { MW; Node } 61-1.7331 \mathrm{MW}\end{array}$ & 0.0704 & 16 & 100 \\
\hline
\end{tabular}

Table 2 and Table 3 show the results of the best solution in 60 trials of implemented methods. The first component value in the multi-objective function of ICOA is 0.3126 , corresponding to 0.0704 MW. Meanwhile, the values for COA and SSA are 0.3129 and 0.3193 , corresponding to $0.0705 \mathrm{MW}$ and $0.0719 \mathrm{MW}$, respectively. In addition, the proposed method is also compared with previously published methods of the power loss reduction in the grid integrated distributed generators system. As shown in Table 3, the power loss with DGs of GA/PSO, SA, BFOA, HSA, IWO, QOTLBO, AGA and ICOA is $0.0811 \mathrm{MW}, 0.0771 \mathrm{MW}, 0.0752 \mathrm{MW}, 0.0868 \mathrm{MW}, 0.0746$ MW, 0.0806 MW, 0.0707 MW and 0.0704 MW, respectively. Explicitly, the power loss of ICOA is the smallest as compared with others. Individually, by installing the optimal location and sizing of PDGs that is proposed from the proposed method, the total power loss can be significantly reduced from $0.2253 \mathrm{MW}$ to $0.0704 \mathrm{MW}$. Thereby, the determination of the suitable installation of PDGs has a great influence on the reduction of power loss in the distribution system. Besides, 
from the results that have been collected for the population and iteration numbers of the methods in Table 3, it has been shown that the population size that is used in the proposed method is significantly lower than the other methods. This indicates that the proposed method only requires a small population size to find a good quality solution. In terms of the iteration number, this number is equal to or higher than the compared methods. As mentioned, this number depends on the results of the survey and it should be large enough to ensure that the convergence is fully completed.

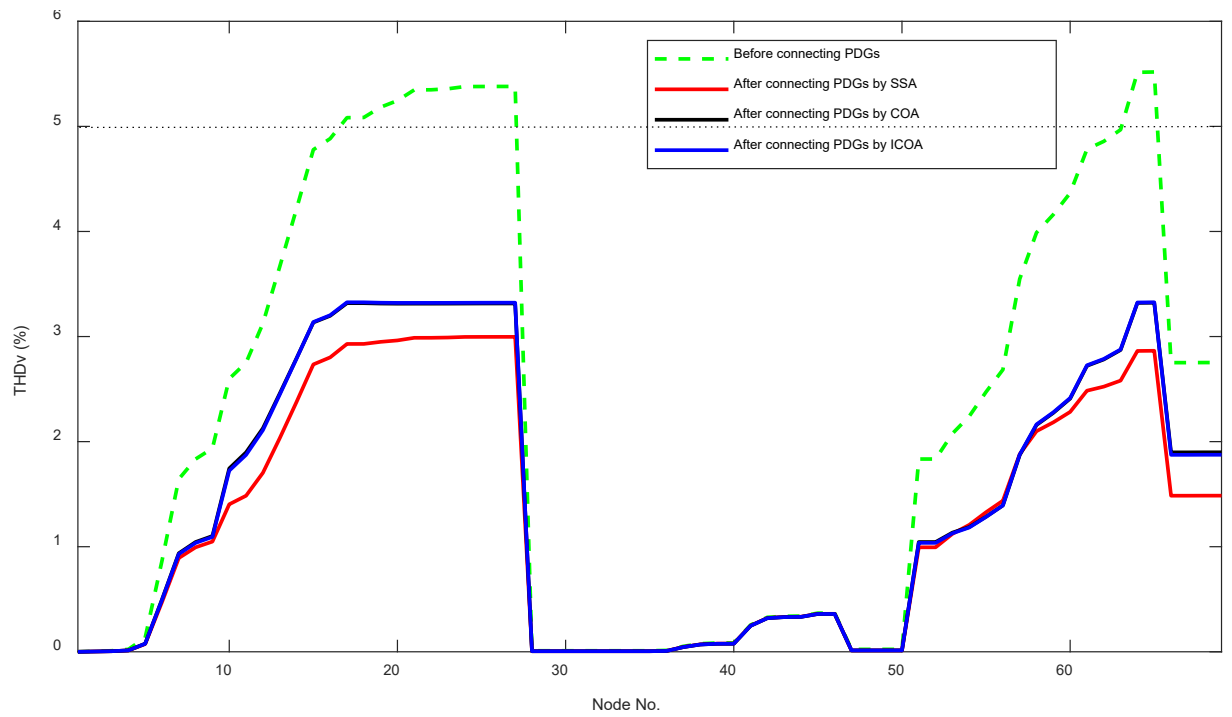

Figure 4. The THDv at each node before and after connecting PDGs

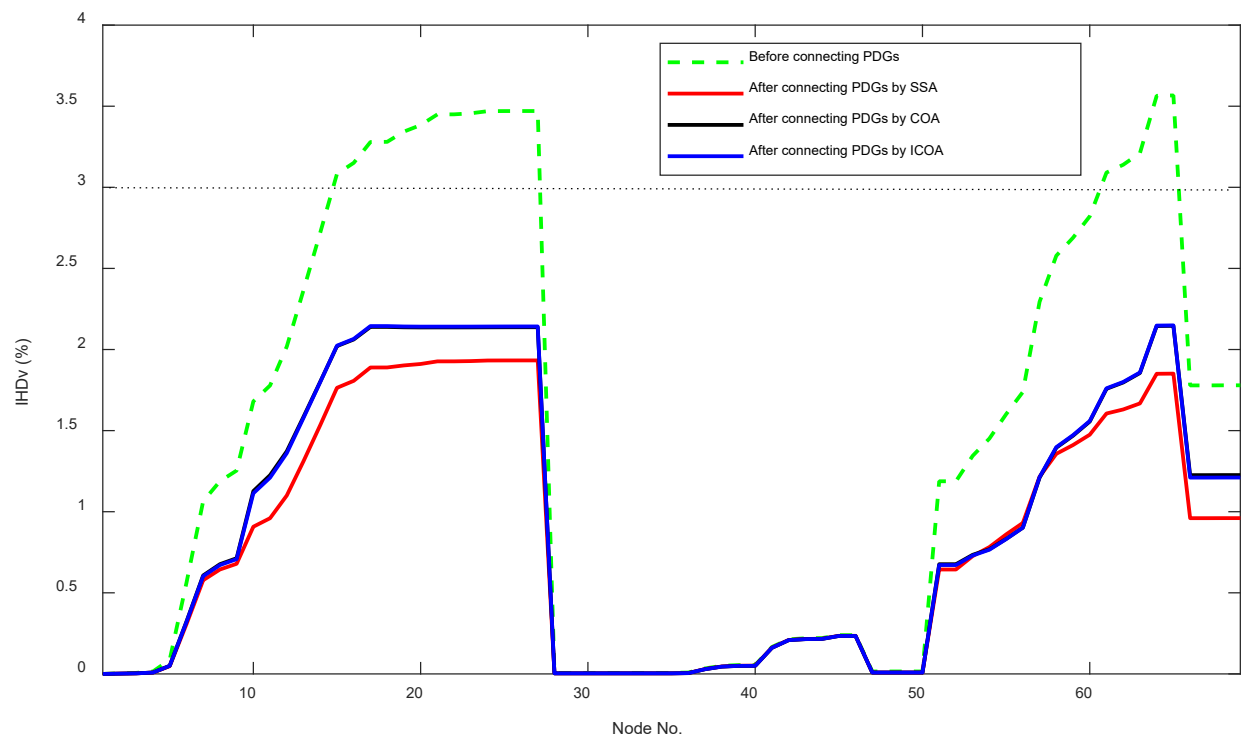

Figure 5. The IHDv at each node before and after connecting PDGs

For the second component value in the multi-objective function, this value of ICOA is 0.9235 and it is the same COA and bigger than SSA, 0.9235 and 0.9027 , respectively. However, the multi-objective function is considered in this study. Therefore, the total objective function value is the decisive value for evaluating the effectiveness of the solution. The total objective function 
values of SSA, COA and ICOA are $0.4360,0.4350$ and 0.4348 respectively. As far as the obtained numerical results, the total objective value of the ICOA is the smallest. In other words, ICOA has a better performance of finding the optimal solution than the two implemented methods.

More details, Figures 4 and 5 show the harmonic distortion profiles in the IEEE 69-node distribution system. As mentioned, five harmonic flows are injected into the system, so the individual voltage harmonic distortion in at each node will have five values, corresponding to five harmonic orders. Therefore, here, the maximum value of individual voltage harmonic distortion at each harmonic order is denoted by $I H D_{V}$. As shown in the Figures, before the connection of PDGs, the maximum values of $T H D_{V}$ and $I H D_{V}$ are equal to $5.5179 \%$ and $3.5665 \%$, respectively. These values exceed the IEEE Std. 519 about the permissible limits of harmonics. However, after the connection of three PDGs, all $T H D_{V}$ and $I H D_{V}$ values at the nodes decreased to the allowable limits as shown in Figures 4 and 5. Typically, the maximum value of $T H D_{V}$ and $I H D_{V}$ which obtained from applying the optimal solution of ICOA is $3.3260 \%$ and $2.1488 \%$, respectively. Obviously, thanks to the proper installation of PDGs, the harmonics of the system are drastically reduced. This shows the influence of integrating PDGs on harmonics. Therefore, the installation of PDGs should be carefully considered to mitigate the harmonics accordingly.

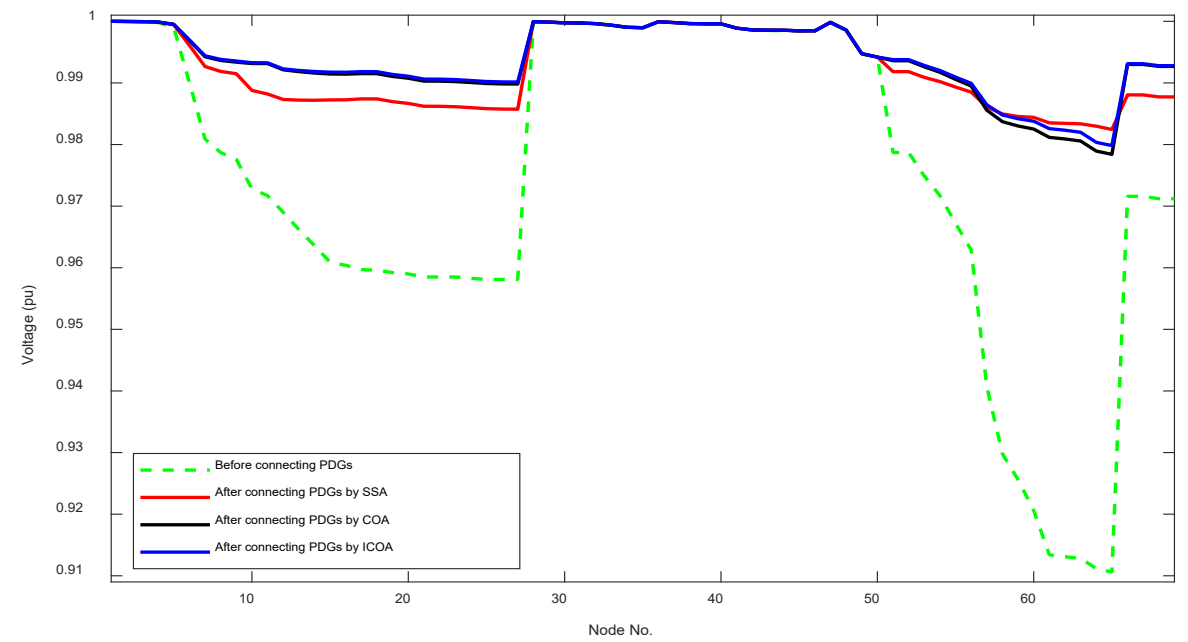

Figure 6. The voltage profile before and after connecting PDGs

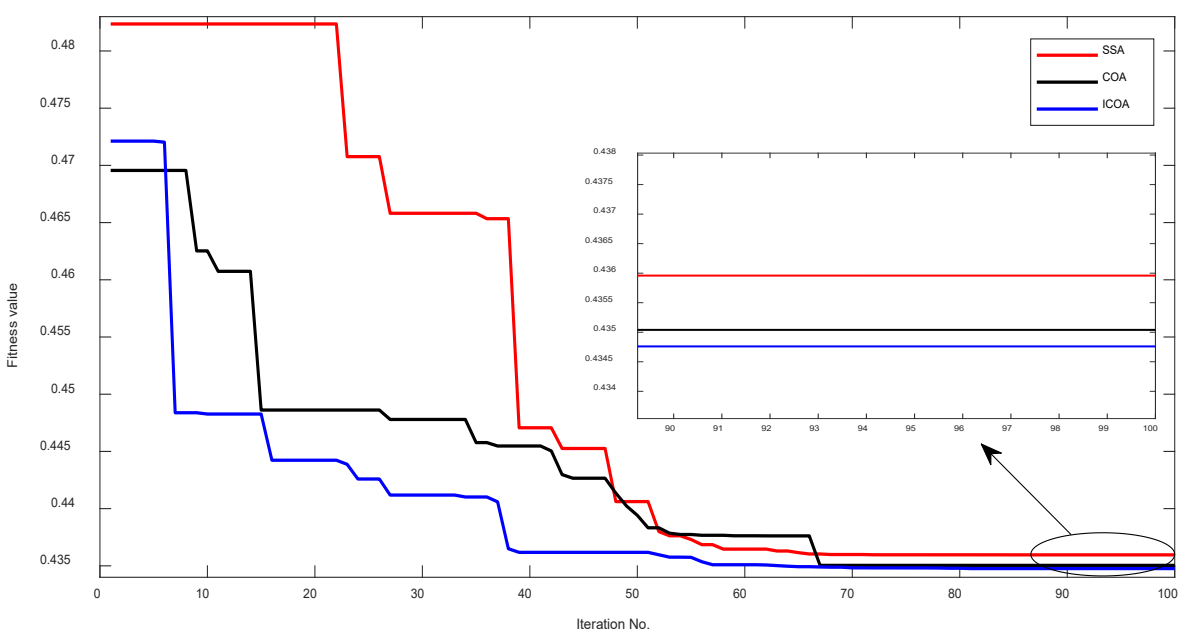

Figure 7. The convergence curves of implemented methods in 100 iterations 
The voltage profiles of the implemented methods before and after integrating the PDGs are shown in Figure 6. Before connection, the minimum node voltage is $0.9106 \mathrm{pu}$. However, when there are properly integrated three PDGs into the system, the voltage profile improved significantly with the smallest node voltage of ICOA of $0.9798 \mathrm{pu}$. Thereby, it shows that the voltage quality has been enhanced thanks to the optimal installation of PDGs into the distribution system.

Figure 7 presents the convergence of these implemented methods. Like the plotted curves, ICOA found a better feasible solution than the others very early, at the $8^{\text {th }}$ iteration. Besides, ICOA also found the best quality solution at the earlier iteration than others, specifically, at the $63^{\text {rd }}$ iteration for ICOA, while at the $70^{\text {th }}$ iteration and at the $68^{\text {th }}$ iteration for COA and SSA, respectively. It indicates that ICOA has better convergence characteristics than compared methods. In short, thanks to suitable improvements in two update equations in COA that ICOA becomes an effective method with the high stability and the fast convergence speed. It is a superior optimization tool in solving the problem of optimizing the location and capacity of PDGs in the distribution system in particular and the optimization problems in general.

\section{Conclusion}

In this research, the improved coyote optimization algorithm is applied to optimize the position and capacity of PDGs in the IEEE 69-node radial distribution system. The main objective function of this work is to minimize power loss and harmonics in the system under the limit constraints of node voltage, branch current and harmonic distortions. Besides, the values of node voltage and power loss are also calculated at the frequency orders to obtain correctly numerical results. The optimal result found by the proposed method reduces power loss significantly from $0.2253 \mathrm{MW}$ to $0.0704 \mathrm{MW}$, corresponding to $68.75 \%$ in the power loss reduction. In addition, $T H D_{V}$ and $I H D_{V}$ also decreased positively from $5.5179 \%$ and $3.5665 \%$ to $3.3260 \%$ and $2.1488 \%$, respectively. This is a clear demonstration of the benefits of properly integrating PDGs in reducing losses as well as harmonics. The found solution from ICOA is also compared to two implemented methods of SSA and COA as well as seven previously published methods of GA/PSO, SA, BFOA, HSA, IWO, QOTLBO and AGA. The obtained results showed that the proposed method is really an efficient, stable and fast convergence method in solving the optimization problem.

\section{Data availability}

The used data of the IEEE 69-node radial distribution system and the detailed information of harmonic flows are taken from Ref. [20] and Ref. [36], respectively.

\section{References}

[1]. A. H. Shojaei, A. A. Ghadimi, M. R. Miveh, F. Mohammadi, F. Jurado, "Multi-objective optimal reactive power planning under load demand and wind power generation uncertainties using $\varepsilon$-constraint method," Applied Sciences, volt. 10, pp.1-30, 2020.

[2]. T. T. Nguyen, L. H. Pham, F. Mohammadi, L. C. Kien, "Optimal scheduling of large-scale wind-hydro-thermal systems with fixed-head short-term model," Applied Sciences, vol. 10, pp. 1-22, 2020.

[3]. A. Naderipour, S. A. Nowdeh, P. B. Saftjani, Z. Abdul-Malek, M. W. B. Mustafa, H. Kamyab, and I. F. Davoudkhani, " Deterministic and probabilistic multi-objective placement and sizing of wind renewable energy sources using improved spotted hyena optimizer," Journal of Cleaner Production, vol. 286, pp. 124941, 2021.

[4]. M. G. Hemeida, A. A. Ibrahim, A. A. A. Mohamed, S. Alkhalaf and A. M. B. El-Dine, "Optimal allocation of distributed generators DG based Manta Ray Foraging Optimization algorithm (MRFO)," Ain Shams Engineering Journal, 2020.

[5]. E. S. Ali, S. M. Abd Elazim and Abdelaziz, "Ant Lion Optimization Algorithm for optimal location and sizing of renewable distributed generations," Renewable Energy, vol. 101, pp. 1311-1324, 2017. 
[6]. T. R. Ayodele, A. S. O. Ogunjuyigbe and O. O. Akinola, "Optimal location, sizing, and appropriate technology selection of distributed generators for minimizing power loss using genetic algorithm," Journal of Renewable Energy, 2015.

[7]. T. Prasetyo, S. Sarjiya, L. M. Putranto," Optimal sizing and siting of PV-based distributed generation for losses minimization of distribution using flower pollination algorithm," In 2019 International Conference on Information and Communications Technology, IEEE, pp. 779-783, 2019.

[8]. S. Ganguly and D. Samajpati, "Distributed generation allocation with on-load tap changer on radial distribution networks using adaptive genetic algorithm," Applied Soft Computing, vol. 59, pp. 45-67, 2017

[9]. S. K. Injeti and N. P. Kumar, "A novel approach to identify optimal access point and capacity of multiple DGs in a small, medium and large scale radial distribution systems," International Journal of Electrical Power \& Energy Systems, vol. 45(1), pp. 142$151,2013$.

[10]. S. Sultana and P. K. Roy, "Multi-objective quasi-oppositional teaching learning based optimization for optimal location of distributed generator in radial distribution systems," International Journal of Electrical Power \& Energy Systems, vol. 63, pp. 534545, 2014.

[11]. E. A. Al-Ammar, K. Farzana, A. Waqar, M. Aamir, A. U. Haq, M. Zahid and M. Batool, "ABC algorithm based optimal sizing and placement of DGs in distribution networks considering multiple objectives," Ain Shams Engineering Journal, volt. 12(1), pp. 697-708, 2021.

[12]. M. Jamil, and A. S. Anees, "Optimal sizing and location of SPV (solar photovoltaic) based MLDG (multiple location distributed generator) in distribution system for loss reduction, voltage profile improvement with economical benefits," Energy, vol. 103, pp. 231-239, 2016.

[13]. R. S. Rao, K. Ravindra, K. Satish and S. V. L. Narasimham, "Power loss minimization in distribution system using network reconfiguration in the presence of distributed generation," IEEE transactions on power systems, vol. 28(1), pp. 317-325, 2012.

[14]. M. Kowsalya, "Optimal size and siting of multiple distributed generators in distribution system using bacterial foraging optimization," Swarm and Evolutionary computation, vol. 15 , pp. 58-65, 2014.

[15]. D. R. Prabha and T. Jayabarathi, "Optimal placement and sizing of multiple distributed generating units in distribution networks by invasive weed optimization algorithm," Ain Shams Engineering Journal, vol. 7(2), pp. 683-694, 2016.

[16]. A. Selim, S. Kamel, and F. Jurado, "Voltage profile improvement in active distribution networks using hybrid WOA-SCA optimization algorithm," In 2018 Twentieth International Middle East Power Systems Conference (MEPCON), IEEE, pp. 1064-1068, December 2018.

[17]. L. F. Grisales-Noreña, D. Gonzalez Montoya and C. A. Ramos-Paja, "Optimal sizing and location of distributed generators based on PBIL and PSO techniques," Energies, vol. 11(4), pp. 1018, 2018.

[18]. M. H. Moradi and M. Abedini, "A combination of genetic algorithm and particle swarm optimization for optimal DG location and sizing in distribution systems," International Journal of Electrical Power \& Energy Systems, vol. 34(1), pp. 66-74, 2012.

[19]. P. Chiradeja, S. Yoomak and A. Ngaopitakkul, "Optimal allocation of multi-DG on distribution system reliability and power losses using differential evolution algorithm," Energy Procedia, vol. 141, pp. 512-516, 2017.

[20]. M. Q. Duong, T. D. Pham, T. T. Nguyen, A. T. Doan and H. V. Tran, "Determination of optimal location and sizing of solar photovoltaic distribution generation units in radial distribution systems," Energies, vol. 12(1), pp. 174, 2019. 
[21]. A. R. Gupta, "Effect of optimal allocation of multiple DG and D-STATCOM in radial distribution system for minimizing losses and THD," In 2017 7th International Symposium on Embedded Computing and System Design, IEEE, pp. 1-5, 2017.

[22]. M. Ashari and O. Penangsang, "Optimal location, size and type of DGs to reduce power losses and voltage deviation considering THD in radial unbalanced distribution systems," In 2016 International Seminar on Intelligent Technology and Its Applications (ISITIA), IEEE, pp. 577-582, 2016.

[23]. A. S. Abubakar, A. A. Olaniyan, A. Ibrahim, S. H. Sulaiman," An improved analytical method for optimal sizing and placement of power electronic based distributed generation considering harmonic limits," In 2019 IEEE PES/IAS PowerAfrica, pp. 122-127, 2019.

[24]. T. T. Nguyen, T. D. Pham, L. C. Kien and L. Van Dai, "Improved coyote optimization algorithm for optimally installing solar photovoltaic distribution generation units in radial distribution power systems," Complexity, 2020.

[25]. A. A. Abou El-Ela, R. A. El-Sehiemy and A. S. Abbas, "Optimal placement and sizing of distributed generation and capacitor banks in distribution systems using water cycle algorithm," IEEE Systems Journal, vol. 12(4), pp. 3629-3636, 2018.

[26]. C. Barutcu, E. Karatepe and M. Boztepe, "Impact of harmonic limits on PV penetration levels in unbalanced distribution networks considering load and irradiance uncertainty," International Journal of Electrical Power \& Energy Systems, vol. 118, pp. 105780, 2020.

[27]. P. Prakash and D. K. Khatod, "Optimal sizing and siting techniques for distributed generation in distribution systems: A review," Renewable and sustainable energy reviews, vol. 57, pp. 111-130, 2016.

[28]. S. Sakar, M. E. Balci, S. H. A. Aleem and A. F. Zobaa, "Integration of large-scale PV plants in non-sinusoidal environments: Considerations on hosting capacity and harmonic distortion limits," Renewable and Sustainable Energy Reviews, vol. 82, pp. 176-186, 2018.

[29]. M. Amini, A. Jalilian and M. R. P. Behbahani, "A new method for evaluation of harmonic distortion in reconFigureuration of distribution network." International Transactions on Electrical Energy Systems, vol. 30(6), pp. 12370, 2020.

[30]. A. Lakum and V. Mahajan, "Optimal placement and sizing of multiple active power filters in radial distribution system using grey wolf optimizer in presence of nonlinear distributed generation," Electric Power Systems Research, vol. 173, pp. 281-290, 2019.

[31]. T.T. Nguyen, F. Mohammadi, "Optimal placement of TCSC for congestion management and power loss reduction using multi-objective genetic algorithm," Sustainability, vol. 12, pp.1-15, 2020.

[32]. Pierezan and L. D. S. Coelho, "Coyote optimization algorithm: a new metaheuristic for global optimization problems," In 2018 IEEE congress on evolutionary computation (CEC), IEEE, pp. 1-8, July 2018.

[33]. M. Milovanović, J. Radosavljević and B. Perović, “A backward/forward sweep power flow method for harmonic polluted radial distribution systems with distributed generation units," International Transactions on Electrical Energy Systems, vol. 30(5), pp. 1-17, 2020.

[34]. T. D. Pham, T. T. Nguyen and B. H. Dinh, "Find optimal capacity and location of distributed generation units in radial distribution networks by using enhanced coyote optimization algorithm," Neural Computing and Applications, pp. 1-29, 2020.

[35]. S. Mirjalili, A. H. Gandomi, S. Z. Mirjalili, S. Saremi, H. Faris and S. M. Mirjalili, "Salp Swarm Algorithm: A bio-inspired optimizer for engineering design problems," Advances in Engineering Software, vol. 114, pp. 163-191, 2017.

[36]. EPRI, Harmonic Spectrum, EPRI, Palo Alto, CA, United States, 2014. https://github.com/tshort/OpenDSS/blob/ master/Test/indmachtest/Spectrum.DSS. 


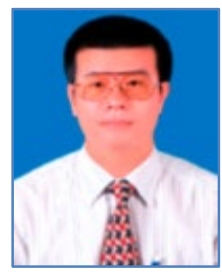

Anh Tuan Doan received his B.E. (2000) at the University of Danang University of Science and Technology (DUT), Vietnam, the Ph.D. (2007) degree in Electrical Engineering, Sait-Peterburg, Russia. His research interests include Power Energy.

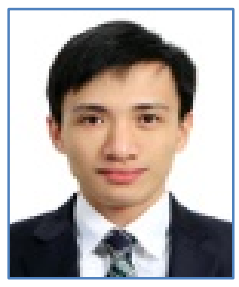

Minh Quan Duong received the B.E. (2008) in Electrical Engineering Department at the University of Danang - University of Science and Technology (DUT), Vietnam and the M.S. (2012) degree in Electrical Engineering at Dongguk University, Seoul, South Korean, and the Ph.D. (2016) degree in Electrical Engineering Politecnico di Milano, Milan, Italia. His research interests include Renewable Energy in Power system and Optimazation, planning.

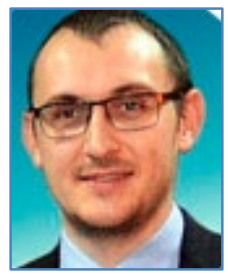

Marco Musetta is Associate Professor in Electrical Engineering, Politecnico di Milano, Milan, Italia - Senior Member IEEE. His research interests include Renewable Energy in Power system. 